\title{
Power System Oscillations Damping Using UPFC Based on an Improved PSO and Genetic Algorithm
}

\author{
Ebrahim Babaei*, Amin Mokari Bolhasan*, Meisam Sadeghi **and Saeid Khani***
}

\begin{abstract}
In this paper, optimal selection of the unified power flow controller (UPFC) damping controller parameters in order to improve the power system dynamic response and its stability based on two modified intelligent algorithms have been proposed. These algorithms are based on a modified intelligent particle swarm optimization (PSO) and continuous genetic algorithm (GA). After extraction of UPFC dynamic model, intelligent PSO and genetic algorithms are used to select the effective feedback signal of the damping controller; then, to compare the performance of the proposed UPFC controller in damping the critical modes of a single-machine infinite-bus (SMIB) power system, the simulation results are presented. The comparison shows the good performance of both presented PSO and genetic algorithms in an optimal selection of UPFC damping controller parameters and damping oscillations.
\end{abstract}

Keywords : UPFC, PSO algorithm, Genetic algorithm, damping controller, power system stability.

\section{Introduction}

Power system stability is defined as the ability of an electric power system, for a given initial operating condition, to regain a state of operating equilibrium after being subjected to a physical disturbance [1]. When large power systems are interconnected through weak tie lines, low frequency oscillations are observed. These oscillations may sustain and grow to cause system separation if no adequate damping is available [2]. Recent development of power electronics devices introduces the use of flexible ac transmission system (FACTS) controllers in power systems. FACTS controllers are capable of controlling the network condition in a very fast manner and this feature of FACTS can be exploited to improve the stability of the power system [3]. In addition, these devices further improve the dynamic performance of the power system in coordination with damping controllers. They can improve system operation because they allow for more accurate control of the power flow, and better and faster control of voltage and system stability. As a result, one of their applications is the damping of power system oscillations, which recently has been attracting the interest of many researchers [3]. The UPFC is

\footnotetext{
* Dept. of Electrical and Computer Engineering, University of Tabriz, Tabriz, Iran. (e-babaei@tabrizu.ac.ir)

** Tabriz Electric Power Distribution Company, Tabriz, Iran. (Maisam_sadeghi@ieee.org)

*** Dept. of Electrical Engineering, Hadishahr branch, Islamic

Azad University, Hadishahr, Iran. (S_khani@ieee.org)

Received 23 July 2011; Accepted 24 October 2011
}

the most versatile FACTS device that has emerged for the control and optimization of power flow in electrical power transmission

systems. Its primary function is to control real and reactive power flow in the line, voltage and current at the UPFC bus. This is achieved by regulating the controllable parameters of the system such as: line impedance, phase angle, and voltage magnitude. Its secondary function is to improve the transient stability and damping of oscillations. Recently, several researchers have been developed for steady state and dynamic models of UPFC. A comprehensive and systematic approach for mathematical modeling of UPFC for steady state and small signal (linearized) dynamic studies has been presented in [4-8].

A modified linearized Heffron-Philips model of a power system installed with UPFC has been presented in [9] and[10].Excellent damping can be achieved via proper controller design for UPFC parameters. By designing a suitable UPFC controller, an effective damping can be achieved. The controllers adjust the UPFC inputs by appropriate processing of the input error signal (speed deviation, $\Delta \omega$ ) and consequently provides an effective damping. Eigenvalue techniques have been commonly used in the design of power system controllers [11-13]. However, these techniques only consider the critical modes in the calculation of feedback gains that can introduce a significant deterioration in damping of the non-critical modes. To avoid such problems, the following important issues must be addressed in the selection of controller gains [14]: 
-Improvement in damping of lightly damped and undamped modes without significantly deteriorating damping criteria of other modes,

-Operation limits of UPFC.

One way for achieving the required performance is to consider controller design as a constrained optimization problem. The constraints include limits of controller gains. The most traditional optimization methods move from one point in the decision hyperspace to another using some deterministic rules. Therefore, probability of getting stuck at a local optimum is a main problem with these methods [14].

The GA is a near global optimization technique that starts with a diverse set (population) of potential solutions (hyperspace vectors). This allows for exploration of many optimums in parallel, lowering the probability of getting stuck at a local optimum [15-17]. This technique is used for optimal design of UPFC based damping controllers.

Recently, PSO technique appeared as a promising algorithm for handling the optimization problems. PSO is a population based stochastic optimization technique, inspired by social behavior of bird flocking or fish schooling. PSO is similar to the continuous GA begins with a random population matrix. Unlike the GA, PSO has no evolution operators such as crossover and mutation [18]. One of the most promising advantages of PSO over GA is its algorithmic simplicity as it uses a few parameters and easy to implement [19].In this paper, the overall model of UPFC in SMIB power system is presented. After that we use PSO for an optimal design of UPFC based damping controllers and then its performance is compared with continuous GA under same condition and with same objective function. The simulation results are used to confirm the ability of the proposed algorithm.

\section{Dynamic Model of UPFC}

Fig. 1 is a SMIB power system installed with a UPFC which consists of an excitation transformer (ET), a boosting transformer (BT), two three-phase GTO based voltage source converters (VSCs) and a dc link capacitor. In this figure, $m_{B}, m_{E}$, and $\delta_{E}, \delta_{B}$ are the amplitude modulation ratio and phase angle of the control signal of each voltage source converter, respectively, which are the input control signals of the UPFC.

The three-phase dynamic differential equations of the UPFC, where transformers resistances and reactances are $\begin{aligned} {\left[\begin{array}{c}\frac{d i_{E a}}{d t} \\ \frac{d i_{E b}}{d t} \\ \frac{d i_{E c}}{d t}\end{array}\right]=} & {\left[\begin{array}{ccc}-\frac{r_{E}}{l_{E}} & 0 & 0 \\ 0 & -\frac{r_{E}}{l_{E}} & 0 \\ 0 & 0 & -\frac{r_{E}}{l_{E}}\end{array}\right]\left[\begin{array}{l}i_{E a} \\ i_{E b} \\ i_{E c}\end{array}\right] } \\ & -\frac{m_{E} v_{d c}}{2 l_{E}}\left[\begin{array}{c}\cos \left(\omega t+\delta_{E}\right) \\ \cos \left(\omega t+\delta_{E}-120^{\circ}\right) \\ \cos \left(\omega t+\delta_{E}+120^{\circ}\right)\end{array}\right]+\left[\begin{array}{ccc}\frac{1}{l_{E}} & 0 & 0 \\ 0 & \frac{1}{l_{E}} & 0 \\ 0 & 0 & \frac{1}{l_{E}}\end{array}\right]\left[\begin{array}{l}v_{E a} \\ v_{E b} \\ v_{E c}\end{array}\right]\end{aligned}$

$$
\left[\begin{array}{c}
\frac{d i_{B a}}{d t} \\
\frac{d i_{B b}}{d t} \\
\frac{d i_{B c}}{d t}
\end{array}\right]=\left[\begin{array}{ccc}
-\frac{r_{B}}{l_{B}} & 0 & 0 \\
0 & -\frac{r_{B}}{l_{B}} & 0 \\
0 & 0 & -\frac{r}{l_{B}}
\end{array}\right]\left[\begin{array}{l}
i_{B a} \\
i_{B b} \\
i_{B c}
\end{array}\right]
$$

$$
\begin{aligned}
& -\frac{m_{B} v_{c c}}{2 l_{B}}\left[\begin{array}{c}
\cos \left(\omega t+\delta_{B}\right) \\
\cos \left(\omega t+\delta_{B}-120\right) \\
\cos \left(\omega t+\delta_{B}+120\right)
\end{array}\right]+\left[\begin{array}{ccc}
\frac{1}{l_{B}} & 0 & 0 \\
0 & \frac{1}{l_{B}} & 0 \\
0 & 0 & \frac{1}{l_{B}}
\end{array}\right]\left[\begin{array}{c}
v_{B a} \\
v_{B b} \\
v_{B c}
\end{array}\right] \\
& \begin{array}{c}
\frac{d v_{d c}}{d t}=-\left[\begin{array}{lll}
\cos \left(\omega t+\delta_{E}\right) & \cos \left(\omega t+\delta_{E}+120\right) & \cos \left(\omega t+\delta_{E}-120\right)
\end{array}\right]\left[\begin{array}{c}
i_{E a} \\
i_{E b} \\
i_{E c}
\end{array}\right] \\
+\frac{m_{B}}{2 C_{d c}}\left[\begin{array}{ccc}
\cos \left(\omega t+\delta_{B}\right) & \cos \left(\omega t+\delta_{B}+120\right) & \cos \left(\omega t+\delta_{B}-120\right)
\end{array}\right]\left[\begin{array}{c}
i_{B a} \\
i_{B b} \\
i_{B c}
\end{array}\right]
\end{array}
\end{aligned}
$$

$$
\begin{aligned}
& {\left[\begin{array}{c}
\frac{d i_{E d}}{d t} \\
\frac{d i_{E q}}{d t} \\
\frac{d i_{E 0}}{d t}
\end{array}\right]=\left[\begin{array}{ccc}
0 & \omega & 0 \\
-\omega & 0 & 0 \\
0 & 0 & 0
\end{array}\right]\left[\begin{array}{l}
i_{E d} \\
i_{E q} \\
i_{E 0}
\end{array}\right]+\left[\begin{array}{ccc}
-\frac{r_{E}}{l_{E}} & 0 & 0 \\
0 & -\frac{r_{E}}{l_{E}} & 0 \\
0 & 0 & -\frac{r_{E}}{l_{E}}
\end{array}\right]\left[\begin{array}{c}
i_{E d} \\
i_{E q} \\
i_{E 0}
\end{array}\right]} \\
& -\frac{m_{E} v_{d c}}{2 l_{E}}\left[\begin{array}{c}
\cos \delta_{E} \\
\sin \delta_{E} \\
0
\end{array}\right]+\left[\begin{array}{ccc}
\frac{1}{l_{E}} & 0 & 0 \\
0 & \frac{1}{l_{E}} & 0 \\
0 & 0 & \frac{1}{l_{E}}
\end{array}\right]\left[\begin{array}{c}
v_{E d} \\
v_{E q} \\
v_{E 0}
\end{array}\right]
\end{aligned}
$$




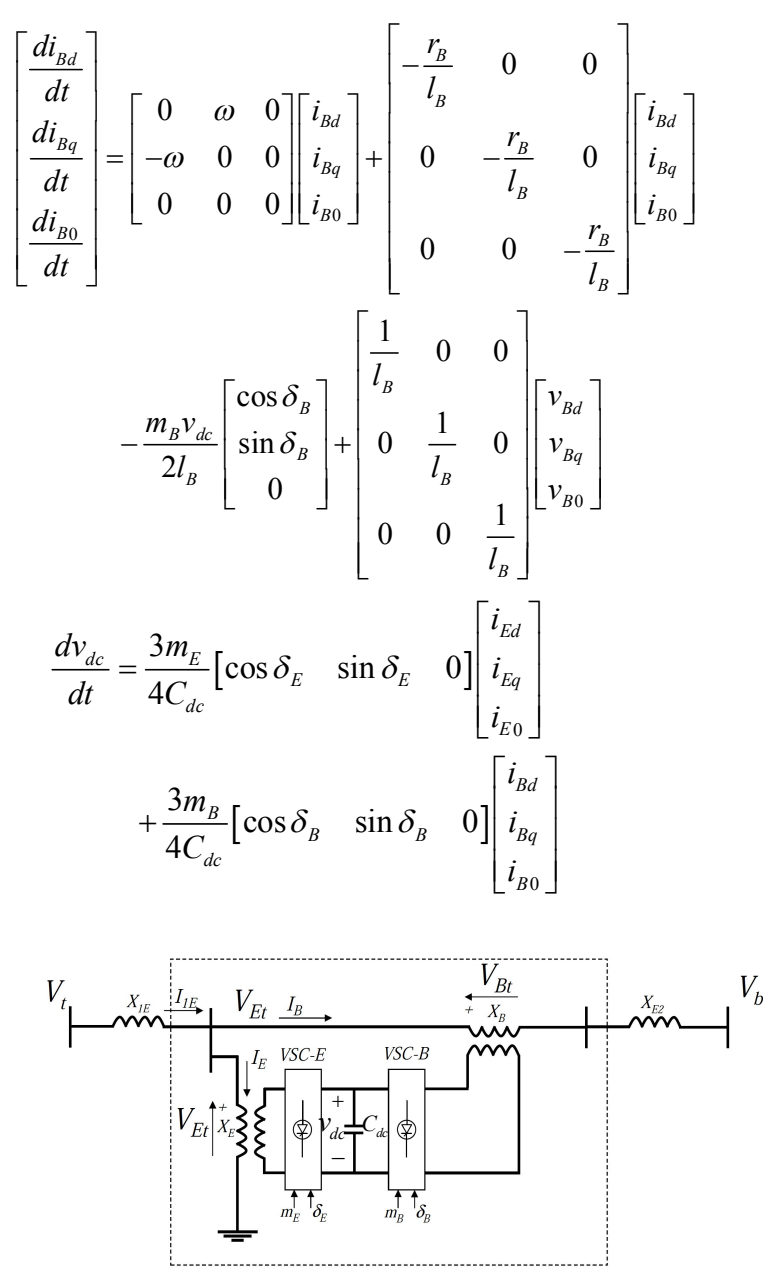

Fig. 1. A UPFC installed in a SMIB power system.

The dynamic model of the UPFC is required in order to study the effect of the UPFC on enhancing the small signal stability of the power system. For the study of power system oscillation stability, the resistance and transient of the transformers of the UPFC can be ignored. So, the above equations can be written as:

$$
\begin{aligned}
& {\left[\begin{array}{c}
v_{E d} \\
v_{E q}
\end{array}\right]=\left[\begin{array}{cc}
0 & -x_{E} \\
x_{E} & 0
\end{array}\right]\left[\begin{array}{l}
i_{E d} \\
i_{E q}
\end{array}\right]+\left[\begin{array}{l}
\frac{m_{E} v_{d c} \cos \delta_{E}}{2} \\
\frac{m_{E} v_{d c} \cos \delta_{E}}{2}
\end{array}\right]} \\
& {\left[\begin{array}{c}
v_{B d} \\
v_{B q}
\end{array}\right]=\left[\begin{array}{cc}
0 & -x_{B} \\
x_{B} & 0
\end{array}\right]\left[\begin{array}{l}
i_{B d} \\
i_{B q}
\end{array}\right]+\left[\begin{array}{l}
\frac{m_{B} v_{d c} \cos \delta_{B}}{2} \\
\frac{m_{B} v_{d c} \cos \delta_{B}}{2}
\end{array}\right]}
\end{aligned}
$$

From Fig. 1:

$$
\begin{gathered}
V_{t}=j x_{1 E} I_{t}+V_{E t} \\
V_{E t}=V_{B t}+j x_{2 E} I_{B}+V_{b}
\end{gathered}
$$

These equations can be expressed in the $d-q$ reference frame as follows:

$$
\begin{gathered}
v_{d t}+j v_{q t}=j x_{1 E}\left(i_{E d}+i_{B d}+j i_{E q}+j i_{B q}\right)+v_{E t d}+j v_{E t q} \\
=x_{q}\left(i_{E q}+i_{B q}\right)+j\left[E_{q}^{\prime}-x_{d}^{\prime}\left(i_{E d}+i_{B d}\right)\right] \\
v_{E t d}+j v_{E t q}=v_{B t d}+j v_{B t q}+j x_{E 2} i_{B d}-x_{E 2} i_{B q} \\
+V_{b} \sin \delta+j V_{b} \cos \delta
\end{gathered}
$$

Using (7) - (12) yields:

$$
\begin{aligned}
& {\left[\begin{array}{c}
i_{E d} \\
i_{B d}
\end{array}\right]=} {\left[\begin{array}{cc}
x_{d}^{\prime}+x_{1 E}+x_{E} & x_{d}^{\prime}+x_{1 E} \\
x_{E} & -x_{B}-x_{E 2}
\end{array}\right]^{-1} } \\
& \times {\left[\begin{array}{c}
E_{q}^{\prime}-\frac{m_{E} v_{d c} \sin \delta_{E}}{2} \\
\frac{m_{B} v_{d c} \sin \delta_{E}}{2}+V_{b} \cos \delta-\frac{m_{E} v_{d c} \sin \delta_{E}}{2}
\end{array}\right] } \\
& {\left[\begin{array}{c}
i_{E q} \\
i_{B q}
\end{array}\right]=\left[\begin{array}{cc}
x_{q}+x_{1 E}+x_{E} & x_{q}+x_{1 E} \\
x_{E} & -x_{B}-x_{E 2}
\end{array}\right]^{-1} } \\
& \times\left[\begin{array}{c}
m_{E} v_{d c} \cos \delta_{E} \\
2
\end{array}\right]
\end{aligned}
$$

The linear model of a SMIB power system is:

$$
\begin{gathered}
g \&=\omega_{0} \Delta \omega \\
\Delta \alpha \&=\left(P_{m}-P_{e}-D \Delta \omega\right) / 2 H \\
E_{q}^{\&}=\left(-E_{q}+E_{f d}\right) / T_{d o}^{\prime} \\
E_{f d}^{\&}=\frac{-E_{f d}+K_{A}\left(v_{r e f}-v_{t}\right)}{T_{A}}
\end{gathered}
$$

where

$$
\begin{gathered}
P_{e}=v_{q t} i_{q t}+v_{d t} i_{d t} \\
v_{q t}=E_{q}^{\prime}-x_{d}^{\prime} i_{d t}, v_{d t}=x_{q} i_{q t}, v_{t}=\sqrt{v_{d t}{ }^{2}+v_{q t}{ }^{2}} . \\
i_{d t}=i_{E d}+i_{B d}, i_{q t}=i_{E q}+i_{B q} \\
E_{q}=E_{q}^{\prime}+\left(x_{d}-x_{d}^{\prime}\right) i_{d t}
\end{gathered}
$$

Let

$$
\begin{aligned}
& {\left[\begin{array}{cc}
x_{q}+x_{1 E}+x_{E} & x_{q}+x_{1 E} \\
x_{E} & -x_{B}-x_{E 2}
\end{array}\right]^{-1}=\left[\begin{array}{ll}
x_{11 q} & x_{12 q} \\
x_{21 q} & x_{22 q}
\end{array}\right]} \\
& {\left[\begin{array}{cc}
x_{d}^{\prime}+x_{1 E}+x_{E} & x_{d}^{\prime}+x_{1 E} \\
x_{E} & -x_{B}-x_{E 2}
\end{array}\right]^{-1}=\left[\begin{array}{ll}
x_{11 d} & x_{12 d} \\
x_{21 d} & x_{22 d}
\end{array}\right]}
\end{aligned}
$$




$$
\begin{aligned}
& \text { Therefore } \\
& {\left[\begin{array}{l}
i_{E d} \\
i_{B d}
\end{array}\right]=\left[\begin{array}{ll}
x_{11 d} & x_{12 d} \\
x_{21 d} & x_{22 d}
\end{array}\right] \times\left[\begin{array}{c}
E_{q}^{\prime}-\frac{m_{E} v_{d c} \sin \delta_{E}}{2} \\
\frac{m_{B} v_{d c} \sin \delta_{E}}{2}+V_{b} \cos \delta-\frac{m_{E} v_{d c} \sin \delta_{E}}{2}
\end{array}\right]} \\
& {\left[\begin{array}{l}
i_{E q} \\
i_{B q}
\end{array}\right]=\left[\begin{array}{ll}
x_{11 q} & x_{12 q} \\
x_{21 q} & x_{22 q}
\end{array}\right] \times\left[\begin{array}{c}
\frac{m_{E} v_{d c} \cos \delta_{E}}{2} \\
\frac{m_{E} v_{d c} \cos \delta_{E}}{2}-V_{b} \sin \delta-\frac{m_{B} v_{d c} \cos \delta_{B}}{2}
\end{array}\right]} \\
& i_{E q}=\frac{x_{11 q} m_{E} \cos \delta_{E} v_{d c}}{2}+\frac{x_{12 q} m_{E} \cos \delta_{E} v_{d c}}{2} \\
& -\frac{x_{12 q} m_{B} \cos \delta_{B} v_{d c}}{2}-x_{12 q} V_{b} \sin \delta \\
& i_{B q}=\frac{x_{21 q} m_{E} \cos \delta_{E} v_{d c}}{2}+\frac{x_{22 q} m_{E} \cos \delta_{E} v_{d c}}{2} \\
& -\frac{x_{22 q} m_{B} \cos \delta_{B} v_{d c}}{2}-x_{22 q} V_{b} \sin \delta \\
& i_{q t}=\frac{\left(x_{11 q}+x_{21 q}+x_{12 q}+x_{22 q}\right) m_{E} \cos \delta_{E} v_{d c}}{2} \\
& -\frac{\left(x_{12 q}+x_{22 q}\right) m_{B} \cos \delta_{E} v_{d c}}{2}-\left(x_{12 q}+x_{22 q}\right) V_{b} \sin \delta
\end{aligned}
$$$$
i_{E d}=x_{11 d} E_{q}^{\prime}-\frac{x_{11 d} m_{E} \sin \delta_{E} v_{d c}}{2}+\frac{x_{21 d} m_{B} \sin \delta_{B} v_{d c}}{2}
$$$$
+x_{12 d} V_{b} \cos \delta-\frac{x_{12 d} m_{E} \sin \delta_{E} v_{d c}}{2}
$$$$
i_{B d}=x_{21 d} E_{q}^{\prime}-\frac{x_{21 d} m_{E} \sin \delta_{E} v_{d c}}{2}+\frac{x_{22 d} m_{B} \sin \delta_{B} v_{d c}}{2}
$$$$
+x_{22 d} V_{b} \cos \delta-\frac{x_{22 d} m_{E} \sin \delta_{E} v_{d c}}{2}
$$$$
\begin{aligned}
i_{d t} & =x_{1 a} E_{q}^{\prime}-\frac{x_{2 a} m_{E} \sin \delta_{E} v_{d c}}{2}+\frac{x_{3 a} m_{B} \sin \delta_{B} v_{d c}}{2} \\
& +x_{4 a} V_{b} \cos \delta
\end{aligned}
$$

The state-space equations of the power system can be represented as:

$$
\begin{aligned}
{\left[\begin{array}{c}
\Delta \delta^{\&} \\
\Delta \alpha^{\&} \\
\Delta E_{q}^{\& t} \\
\Delta E_{f d}^{\&} \\
\Delta \nu_{d c}^{\&}
\end{array}\right] } & {\left[\begin{array}{ccccc}
0 & \omega_{b} & 0 & 0 & 0 \\
-\frac{K_{1}}{M} & -\frac{D}{M} & -\frac{K_{2}}{M} & 0 & -\frac{K_{p d}}{M} \\
-\frac{K_{4}}{T_{d o}^{\prime}} & 0 & -\frac{K_{3}}{T_{d o}^{\prime}} & \frac{1}{T_{d o}^{\prime}} & -\frac{K_{q d}}{T_{d o}^{\prime}} \\
-\frac{K_{A} K_{5}}{T_{A}} & 0 & -\frac{K_{A} K_{6}}{T_{A}} & -\frac{1}{T_{A}} & -\frac{K_{A} K_{v d}}{T_{A}} \\
K_{7} & 0 & K_{8} & 0 & -K_{9}
\end{array}\right]\left[\begin{array}{c}
\Delta \delta \\
\Delta \omega \\
0 E_{q}^{\prime} \\
\Delta E_{f d} \\
\Delta V_{d c}
\end{array}\right] } \\
& +\left[\begin{array}{cccc}
0 & 0 & 0 & 0 \\
-\frac{K_{p e}}{M} & -\frac{K_{p \delta e}}{M} & -\frac{K_{p b}}{M} & -\frac{K_{p \delta b}}{M} \\
-\frac{K_{q e}}{T_{d o}^{\prime}} & -\frac{K_{q \delta e}}{T_{d o}^{\prime}} & -\frac{K_{q b}}{T_{d o}^{\prime}} & -\frac{K_{q \delta b}}{T_{d o}^{\prime}} \\
-\frac{K_{A} K_{v e}}{T_{A}} & -\frac{K_{A} K_{v \delta e}}{T_{A}} & -\frac{K_{A} K_{v b}}{T_{A}} & -\frac{K_{A} K_{v \delta b}}{T_{A}} \\
K_{d c e} & K_{d c \delta e} & K_{d c b} & K_{d c \delta b}
\end{array}\right]\left[\begin{array}{l}
\Delta m_{E} \\
\Delta \delta_{E} \\
\Delta m_{B} \\
\Delta \delta_{B}
\end{array}\right]
\end{aligned}
$$

where $\Delta m_{E}, \Delta m_{B}, \Delta \delta_{E}, \Delta \delta_{B}$ are the deviation of input control signals of the UPFC and explained previously. The modified Heffron -Philips model for the system with UPFC is shown in Fig.2.

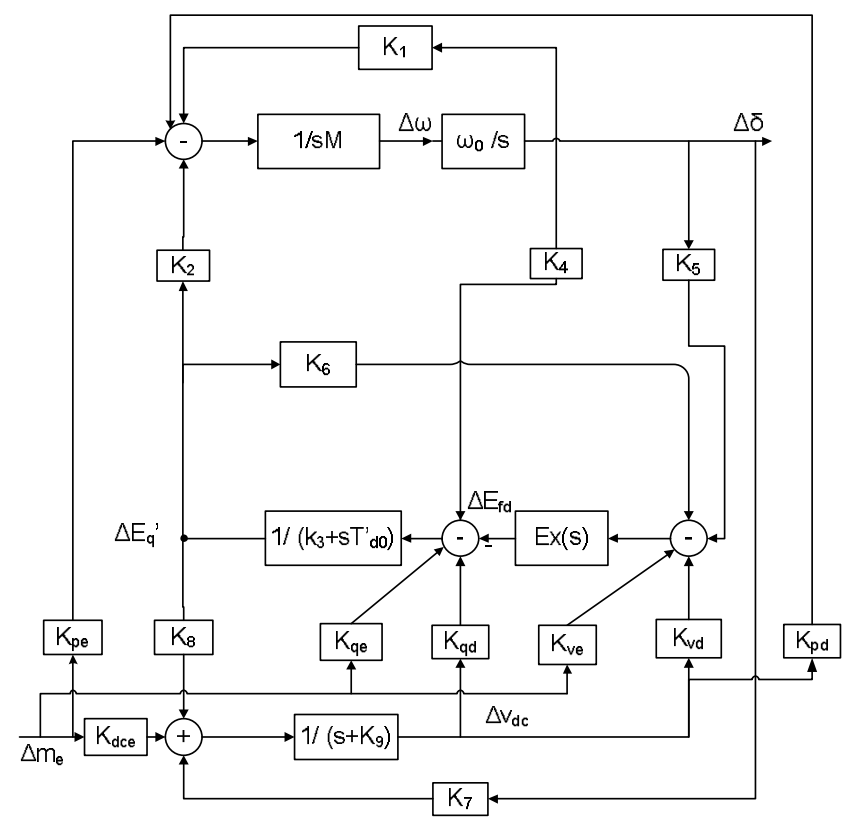

Fig. 2. Modified Heffron-Phillips transfer function model.

\section{Damping Controller Design}

In order to improve the damping ratio of a machine torque, a modified damping controller must be designed. A sample block diagram of the UPFC damping controller is illustrated 
in Fig. 3 that contains of three main parts: gain block, signal filter, and phase compensators.

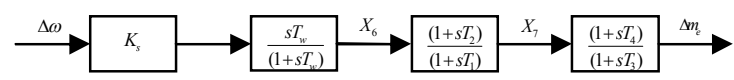

Fig. 3. Simplified UPFC damping controller block diagram.

The signal filter is a high pass filter that modifies the UPFC input signal and prevents steady changes in active power. Also $T_{w}$ should have a large value but this amount is not critical and may be in the range of 1 to 20 seconds.

With application of the above dynamic controller, the number of state variables increases from 5 to 8 . In this paper, two main intelligent algorithms (PSO and GA) have been used to find the optimized values of compensator block's gain and phase parameters. Then their capability in controlling and damping of oscillations have been investigated and compared.

In order to calculate the closed loop state matrix with damping controller two state variables $\left(X_{6}\right.$ and $\left.X_{7}\right)$ have been chosen as shown in Fig. 3. According to Fig. 3:

$$
\begin{aligned}
& \frac{X_{6}}{\Delta \omega}=\frac{K_{s} s T_{w}}{1+s T_{w}} \\
& X_{6}+T_{w} X_{6}^{\&}=K_{s} T_{w} \Delta \&
\end{aligned}
$$

Therefore

$$
X_{6}^{\&}=\frac{K_{s} T_{w} \Delta \alpha_{L}-X_{6}}{T_{w}}
$$

By replacing $\Delta \&$ form (33) in (35), 姑 can be obtained as follows:

$$
\begin{aligned}
\not x_{6}^{\&} & =K_{s}\left(-\frac{K_{1}}{M} \Delta \delta-\frac{D}{M} \Delta \omega-\frac{K_{2}}{M} \Delta E_{q}^{\prime}-\frac{K_{p d}}{M} \Delta V_{d c}-K_{p e} \Delta m_{E}\right) \\
& -\frac{X_{6}}{T_{w}}
\end{aligned}
$$

$X_{7}^{\&}$ and $\Delta n_{E} \&_{E}$ can be also obtained from the mentioned above equations. Finally, with adding these three state variables into state matrix, the closed loop matrix will be $[8 \times 8]$.Now, in order to determine the optimized values of damping controller parameters $\left(T_{1}, T_{2}, T_{3}, T_{4}\right.$ and $\left.K_{s}\right)$ two intelligent algorithms, modified PSO and GA have been used as described in the following.

\subsection{Application of PSO}

The PSO is a swarm intelligence class method proposed by Kennedy and Eberhart (1995).The PSO is a probabilistic optimized technique based on swarm behavior which is resulted from birds or fishes swarm behaviors. In the PSO, each potential solution is referred to as a particle and each set of particles composes a population. Each particle maintains the position associated with the best fitness it ever experiences in a personal memory called $p_{\text {best }}$. In addition, the position associated with the best value obtained so far by any particle is called $g_{\text {best }}$. In any iteration, the $p_{\text {best }}$ and $g_{\text {best }}$ values are updated and each particle modifies its velocity to move toward them stochastically. This concept can be formulated as

$$
\begin{gathered}
v_{i}^{t+1}=w \cdot v_{i}^{t}+c_{1} \cdot\left(\text { pbest }_{i}-x_{i}^{t}\right)+c_{2} r_{2} \cdot\left(\text { gbest }-x_{i}^{t}\right) \\
x_{i}^{t+1}=x_{i}^{t}+v_{i}^{t+1}, i=1,2, \ldots, n
\end{gathered}
$$

where $v$ is the particle velocity, $x$ is the particle position, $n$ is the number of particles, $t$ is the number of iterations, $w$ is the inertia weight factor, $c_{1}$ and $c_{2}$ are the cognitive and social acceleration factors, respectively, and $r_{1}$ and $r_{2}$ are the uniformly distributed random numbers in the range $(0,1)$.

Fig. 4 shows the flow chart of the PSO algorithm for the coordinated design of the power system stabilizer (PSS) and UPFC problem [20].

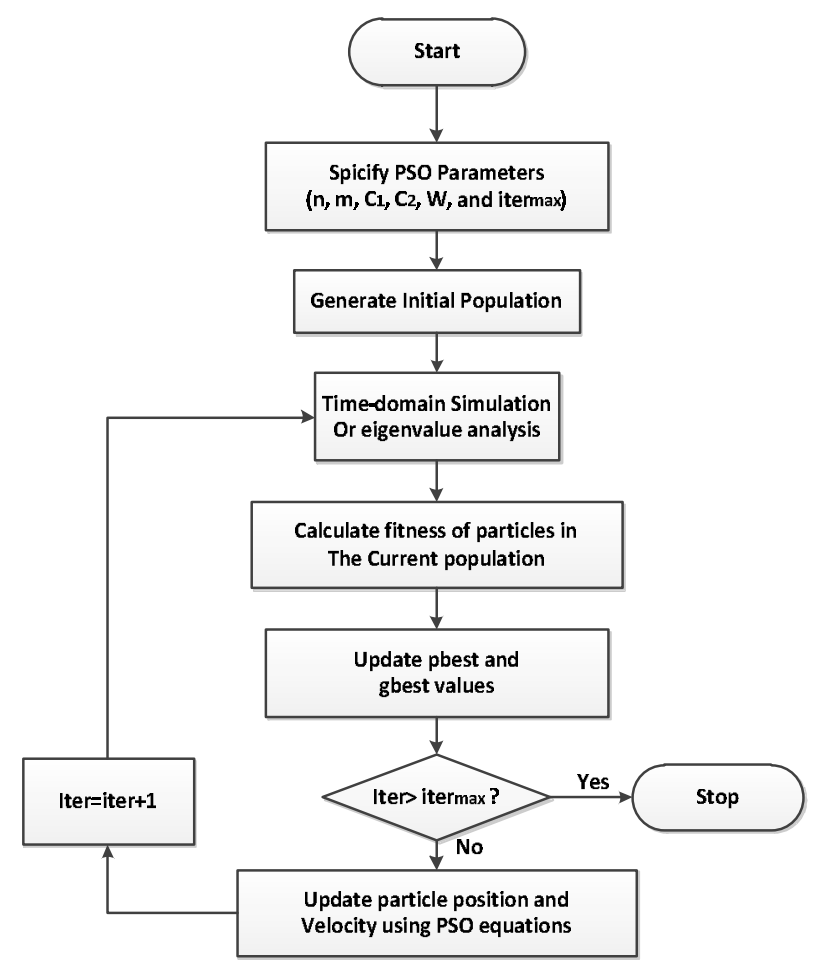

Fig. 4. Flow chart of the (PSO) algorithm for coordinated design of the (PSS) and UPFC problem. 


\subsection{Application of genetic algorithm}

The GA uses the principle of natural evolution and population genetics to search and arrive at a high quality near global solution [15-17]. The required design variables are encoded into a binary string as a set of genes corresponding to chromosomes in biological systems. Unlike the traditional optimization techniques that require one starting point, they use a set of points as the initial condition. Each point is called a chromosome and a group of chromosomes are called a population [17].In this paper, the continuous GA has been used to find the optimized damping controller parameters. The continues GA is faster and more accurate than binary GA and also this algorithm uses less memory.

\subsection{Objective Function}

Main goal of control system is increasing of critical modes damping without destroying uncritical modes. Real part of closed-loop eigenvalues indicates the critical modes of system and must be inserted in goal function [21]. As a result, the goal function can be expressed as below:

$$
\min C=\sum_{i=1}^{n} P^{\lambda i}
$$

where $P^{\lambda i}$ is given as:

$$
P^{\lambda i}=\left\{\begin{array}{cc}
0 & \text { if } \sigma^{i} \leq \sigma^{i d} \\
\sigma^{i}-\sigma^{i d} & \text { otherwise }
\end{array}\right\}
$$

where $\sigma^{i}$ and $\sigma^{i d}$ are modified and desired values of real part of i-th eigenvalue.

\section{Simulation Results}

In this section, the SMIB power system installed with UPFC (Fig. 1) is investigated. Digital simulation has been carried out with Modified Heffron Phillips model in MATLAB environment. The simulation result of the Modified Heffron Phillips model with four different input control signals under different load conditions in mechanical power input is considered for analysis.

Here, we use one of input signals $\left(m_{E}\right)$ which is controlled by PSS parameters obtained from PSO or GA. First, the open-loop eigenvalues that obtained from equations (15)-(18) are calculated and given in Table I.
Table 1. System eigenvalues without damping controller

\begin{tabular}{|l|l|l|l|}
\hline \multicolumn{4}{|c|}{ Open loop eigenvalues } \\
\hline$-0.0166 \pm 2.3704 \mathrm{i}$ & -85.3113 & -14.8888 & -0.0092 \\
\hline
\end{tabular}

Now, in order to move the eigenvalues to desired place, the system will be equipped with damping controller using PSO and GA algorithms.

\subsection{Closed-Loop System using Modified PSO Algorithm}

The closed-loop eigenvalues of system when PSO algorithm is used to find the damping controller parameters are shown in Table II. Because the objective function of PSO algorithm has been modified, the parameters have been founded so faster. Damping controller parameters based on UPFC are given as below:

$$
\begin{aligned}
& K_{s}=19.1937, T_{1}=0.9544, \quad T_{2}=2.6880, \\
& T_{3}=1.1145, T_{4}=0.9418
\end{aligned}
$$

Table 2. Closed-loop eigenvalues using PSO algorithm

\begin{tabular}{|c|c|c|c|c|c|c|}
\hline \multicolumn{7}{|c|}{ Closed-loop eigenvalues } \\
\hline $\begin{array}{r}-0.8894 \\
\pm 2.46 \mathrm{i}\end{array}$ & -85.29 & -14.99 & -0.009 & -0.08 & -0.048 & -0.05 \\
\hline
\end{tabular}

\subsection{Closed-Loop System using Genetic Algorithm}

Table III. illustrates the closed-loop eigenvalues of system when GA has been used. Damping controller parameters based on UPFC which are specified by GA are given as below:

$$
\begin{aligned}
& K_{s}=18.5666, T_{1}=0.715139, \quad T_{2}=0.17221, \\
& T_{3}=0.222375, T_{4}=1.52679
\end{aligned}
$$

Table 3. Closed-loop eigenvalues using GA algorithm

\begin{tabular}{|c|c|c|c|c|c|c|}
\hline \multicolumn{7}{|c|}{ Closed-loop eigenvalues } \\
\hline $\begin{array}{c}-0.36468 \\
\pm 0.8884 \mathrm{i}\end{array}$ & -84.72 & -37.83 & -13.65 & -1.20 & -0.087 & -0.0094 \\
\hline
\end{tabular}

A sample step distortion has been exerted on the input of system block diagram at $\mathrm{t}=1 \mathrm{sec}$ and simulated. Fig. 5.shows the simulation result of the power system implemented by MATLAB according to the dynamic model. As shown in Fig. 5.the intelligent algorithm based on damping controller of UPFC can noticeably damp the speed variation and improve the dynamic response of the system. 


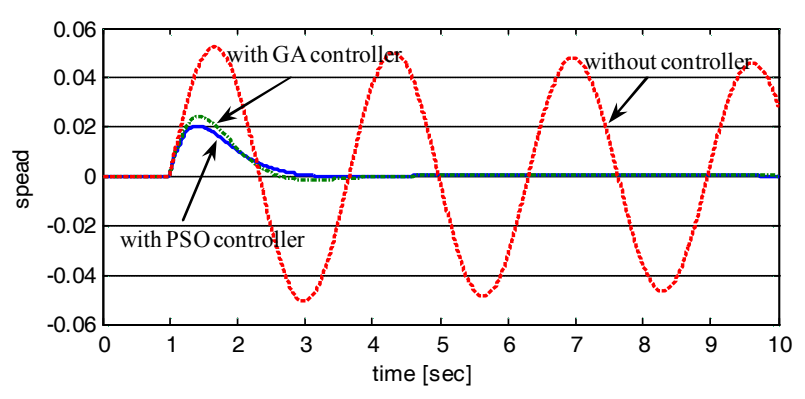

Fig. 5. Speed variation without and with PSO or GA based controllers.

\section{Conclusion}

This paper presents the overall model development of UPFC in SMIB power system. The model has been used to design the optimal damping controller with the concept of eigenvalue assignment technique. In order to find the optimized values of the controller parameters, two main modified intelligent algorithms (PSO and GA) have been used. Using these two modified intelligent algorithms, the damping ratio is increased as well as the variations are omitted in one and half of a cycle. In this paper, we assume one of the UPFC inputs $\left(m_{e}\right)$ as an output for damping controller which is optimized by both PSO and GA. It is observed that PSO algorithm, in addition to its simplification and high speed calculations, has better results to transfer the unstable modes to the left side of imaginary axes and oscillation damping than GA. The proposed design also minimizes deterioration of noncritical modes while keeping constraints within the permissible limits.

\section{Acknowledgements}

Parameters of used single-machine infinite-bus power system:

$$
\begin{array}{lllll}
M=8 & D=0 & T_{d 0}^{\prime}=5 & X_{d}=0.973 & X_{q}=0.55 \\
X_{d}^{\prime}=100 & T_{A}=0.01 & X_{1 E}=0.3 & X_{E 2}=0.3 & X_{E}=0.03 \\
X_{B}=1.05 & V_{t}=1 & \delta_{0}=15.87^{\circ} & V_{d c 0}=1 & C_{d c 0}=1
\end{array}
$$

\section{References}

[1] P. Kundur, J. Paserba, V. Ajjarapu, G. Andersson, A. Bose, C. Canizares, N. Hatziargyriou, D. Hill, A. Stankovic, C. Taylor, V.T. Cutsem, and V. Vittal, "Definition and classification of power system stability IEEE/CIGRE joint task force on stability terms and definitions," IEEE Trans. Power Syst., vol. 19, no. 3, pp. 1387-1401, Aug. 2004.
[2] . P. Kundur, "Power system stability and control," McGrawHill, 1994.

[3] N.G. Hingorani and L. Gyugyi, "Understanding FACTS: concepts and technology of flexible AC transmission systems," IEEE Press, New York, 2000.

[4] H.F. Wang, "A unified model for the analysis of FACTS devices in damping power system oscillations - Part III: unified power flow controller," IEEE Trans. Power Del., vol. 15, no. 3; pp. 978-983, 2000.

[5] B.T. Ooi, M. Kazerani, R. Marceau, Z. Wolanki, F.D. Galiana, D. McGillis, and G. Joos, "Mid-point sitting of FACTS devices in transmission lines," IEEE Trans. Power Del., vol. 12, no. 4, pp. 1717-1722, October 1997.

[6] M. Noroozian, L. Angquist, M. Ghandari, and G. Anderson, "Use of UPFC for optimal power flow control," IEEE Trans. Power Syst., vol. 12, no. 4, pp. 1629-1634, 1997.

[7] A. Nabavi-Niaki and M.R. Iravani, "Steady-state and dynamic models of unified power flow controller (UPFC) for power system studies," IEEE Trans. Power Syst., vol. 11, no. 4, p 1937, Nov. 1996.

[8] K.S. Smith, L. Ran, and J. Penman, "Dynamic modeling of a unified Power flow controller," IEE Proceedings-C, vol. 144, no. 1, p. 7, Jan. 1997.

[9] H.F. Wang, "Damping function of unified power flow controller," IEE Proceedings-C, vol. 146, no. 1, p. 81, Jan. 1999.

[10] H.F. Wang and F.J. Swift, "A unified model for the analysis of FACTS devices in damping power system oscillations Part I: Single-machine infinite-bus power systems," IEEE Trans. Power Del., vol. 12, no. 2, pp. 941-946, April 1997.

[11] E.V. Larsen, J.J. Sanchez-Gasca, J.H. Chow, "Concepts for design of FACTS controllers to damp power swings," IEEE Trans. Power Del., vol. 10, no. 2, pp. 948-956, May 1995.

[12] X.R. Chen, N.C. Pahalawaththa, U.D. Annakkage, C.S. Kumble, "Design of TCSC controllers to damp power swings by using eigenvalue analysis method, "IEEE/KTH Stockholm Power Tech. Conference, Stockholm, Sweden, pp.268-275, June 18-22 1995.

[13] E. Babaei, M. Hasan Nia Khabiri, "Determination of eigenvalues sensitivity-Based filter parameters using genetic algorithm," in Proc. $19^{\text {th }}$ Iranian Conference on Electrical Engineering, 2011, Tehran, Iran (in Persian).

[14] E. Babaei and V. Hosseinnezhad, "A QPSO based parameters tuning of the conventional power system stabilizer," in Proc. IPEC, 2010, Singapore, pp. 467-471.

[15] D.E. Goldberg, "Genetic algorithms in search, optimization, and machine learning," Addison-Wesley, New York, 1989.

[16] E. Babaei, S. Galvani, and M. Ahmadi Jirdehi, "Design of robust power system stabilizer based on PSO," in Proc. 2009 IEEE Symposium on Industrial Electronics and Applications (ISIEA), 2009, Kuala Lumpur, Malaysia, vol. 1, pp. 325-330.

[17] L. Davis, "Handbook of genetic algorithms," Van Nostrand Reinhold, New York, 1991.

[18] R.L. Haupt and S.E. Haupt, "Practical genetic algorithms," John Wiley \& Sons INC, 2004.

[19] PSO http://www.swarmintelligence.org/tutorials.php Tutorial 
[20] A. Rezazadeh, M. Sedighzadeh, and A. Hasaninia, "Coordination of PSS and TCSC controller using modified particle swarm optimization algorithm to improve power system dynamic performance," Journal of Zhejiang University-SCIENCE C (Computers \& Electronics).

[21] S. Khani, M. Sadeghi, and S.H. Hosseini, "Optimal design of SSSC damping controller to improve power system dynamic stability using modified intelligent algorithms," in Proc. PEDSTC, 2010, Iran, pp. 393-297.

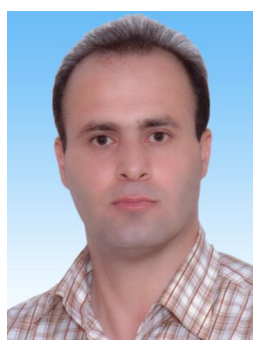

Ebrahim Babaei was born in Ahar, Iran in 1970. He received his B.S. and M.S. in Electrical Engineering from the Department of Engineering, University of Tabriz, Tabriz, Iran, in 1992 and 2001, respectively, graduating with first class honors. He received his Ph.D. in Electrical Engineering from the Department of Electrical and Computer Engineering, University of Tabriz, Tabriz, Iran, in 2007. In 2004, he joined the Faculty of Electrical and Computer Engineering, University of Tabriz. He was an Assistant Professor from 2007 to 2011 and has been an Associate Professor since 2011. $\mathrm{He}$ is the author of more than 120 journal and conference papers. His current research interests include the analysis and control of power electronic converters, matrix converters, multilevel converters, FACTS devices and power system dynamics.

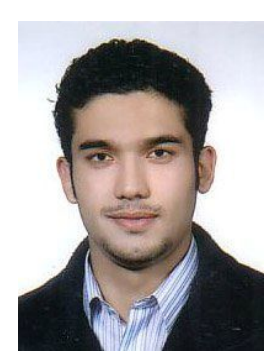

Amin Mokari Bolhasan was born in Tabriz, Iran, in 1988. He received the B.Sc. degree in electrical engineering from the University of Tabriz, Iran, in 2010 and is currently pursuing M.Sc. degree in power system electrical engineering in university of Tabriz, Iran. His current research interests include power system dynamics, power system planning, system protection and also distributed generation.

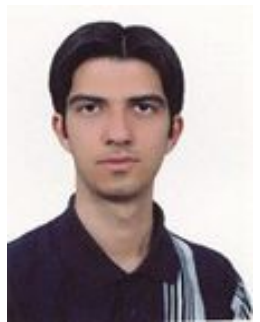

Meisam Sadeghi (S'10) was born in Maku, Iran, in 1985. He received the B.S and M.S. (Hons.) degrees in electrical engineering from the University of Tabriz, Tabriz, in 2008 and 2011, respectively. He is author or coauthor of more than 16 journal and conference papers. His current research interests include power electronic circuits, multilevel converters, power quality, D-STATCOM, dynamic voltage restorer and also power system dynamics, power system planning.

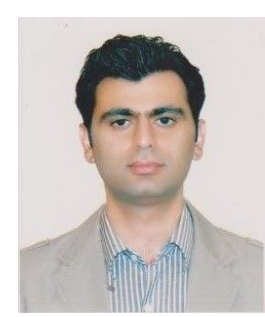

Saeid Khani was born in Tabriz, Iran, 1984. He received his B.Sc. degree in electrical engineering from the University of Zanjan, Iran and M.Sc. degree in electrical engineering from the University of Tabriz, Iran in 2007 and 2011 respectively. His research interests include Power Electronic Converters and Power Electronic Application in Renewable Energy Systems, Power Quality Enhancement and FACTS. 